\title{
Generalized definition of time delay in scattering theory
}

\author{
Christian Gérard and Rafael Tiedra de Aldecoa \\ Département de mathématiques, Université de Paris XI, \\ 91405 Orsay Cedex France \\ E-mails: christian.gerard@math.u-psud.fr and rafael.tiedra@math.u-psud.fr
}

\begin{abstract}
We advocate for the systematic use of a symmetrized definition of time delay in scattering theory. In two-body scattering processes, we show that the symmetrized time delay exists for arbitrary dilated spatial regions symmetric with respect to the origin. It is equal to the usual time delay plus a new contribution, which vanishes in the case of spherical spatial regions. We also prove that the symmetrized time delay is invariant under an appropriate mapping of time reversal. These results are also discussed in the context of classical scattering theory.
\end{abstract}

\section{Introduction}

This paper is devoted to the definition of time delay (in terms of sojourn times) in scattering theory. Its purpose is to advocate for the systematic use of a symmetrized definition of time delay. Our main arguments supporting this point of view are the following:

(A) Symmetrized time delay generalizes to multichannel-type scattering processes. Usual time delay does not.

(B) In two-body scattering processes, symmetrized time delay and usual time delay are equal.

(C) In two-body scattering processes, symmetrized time delay does exist for arbitrary dilated spatial regions symmetric with respect to the origin (usual time delay does exist only for spherical spatial regions [9]). It is equal to the usual time delay plus a new contribution, which vanishes in the case of spherical spatial regions.

(D) Symmetrized time delay is invariant under an appropriate mapping of time reversal. Usual time delay is not.

Our purpose in this paper is to give the precise meaning and the proof of these statements. 
Let us first recall the usual and the symmetrized definition of time delay for a twobody scattering process in $\mathbb{R}^{d}, d \geq 1$. Consider a bounded open set $\Sigma$ in $\mathbb{R}^{d}$ containing the origin and the dilated spatial regions $\Sigma_{r}:=\{r x \mid x \in \Sigma\}, r>0$. Let $H_{0}:=-\frac{1}{2} \Delta$ be the kinetic energy operator in $\mathcal{H}:=\mathrm{L}^{2}\left(\mathbb{R}^{d}\right)$ and let $H$ be a selfadjoint perturbation of $H_{0}$ such that the wave operators $W^{ \pm}:=\mathrm{s}-\lim _{t \rightarrow \pm \infty} \mathrm{e}^{1 t H} \mathrm{e}^{-1 t H_{0}}$ exist and are complete (so that the scattering operator $S:=\left(W^{+}\right)^{*} W^{-}$is unitary). Then one defines for some states $\varphi \in \mathcal{H}$ and $r>0$ two sojourn times, namely:

$$
T_{r}^{0}(\varphi):=\int_{-\infty}^{\infty} \mathrm{d} t \int_{x \in \Sigma_{r}} \mathrm{~d}^{d} x\left|\left(\mathrm{e}^{-1 t H_{0}} \varphi\right)(x)\right|^{2}
$$

and

$$
T_{r}(\varphi):=\int_{-\infty}^{\infty} \mathrm{d} t \int_{x \in \Sigma_{r}} \mathrm{~d}^{d} x\left|\left(\mathrm{e}^{-1 t H} W^{-} \varphi\right)(x)\right|^{2} .
$$

If the state $\varphi$ is normalized the first number is interpreted as the time spent by the freely evolving state $\mathrm{e}^{-1 t H_{0}} \varphi$ inside the set $\Sigma_{r}$, whereas the second one is interpreted as the time spent by the associated scattering state $\mathrm{e}^{-1 t H} W^{-} \varphi$ within the same region. The (usual) time delay of the scattering process with incoming state $\varphi$ for $\Sigma_{r}$ is defined as

$$
\tau_{r}^{\text {in }}(\varphi):=T_{r}(\varphi)-T_{r}^{0}(\varphi) .
$$

For a suitable initial state $\varphi$, a sufficiently short-ranged interaction and $\Sigma_{r}$ spherical, the limit of $\tau_{r}^{\text {in }}(\varphi)$ as $r \rightarrow+\infty$ exists and is equal to the expectation value in the state $\varphi$ of the Eisenbud-Wigner time delay operator [2, 3]. For multichannel-type scattering processes such as $N$-body scattering [10, 5, 7], scattering with dissipative interactions [6], step potential scattering [4] and scattering in waveguides [11], a definition such as (1.1) for time delay is inappropriate. In such cases time delay of the form (1.1) do not admit a limit due to the "non-conservation" of the kinetic energy. Therefore one has to modify the definition (1.1) by replacing the free sojourn time $T_{r}^{0}(\varphi)$ with the effective free sojourn time $\frac{1}{2}\left[T_{r}^{0}(\varphi)+T_{r}^{0}(S \varphi)\right]$ (see e.g. [7] Sec. V.(a)] or [11] Sec. 1] for details). In two-body scattering, this modified (symmetrized) time delay takes the form:

$$
\tau_{r}(\varphi):=T_{r}(\varphi)-\frac{1}{2}\left[T_{r}^{0}(\varphi)+T_{r}^{0}(S \varphi)\right] .
$$

We stress that this effective time delay generalises to multichannel-type scattering processes (i.e. its multichannel counterpart admits a limit as $r \rightarrow+\infty$ ), which is not the case for the time delay (1.1.

In Section 4.2 we prove that the time delay $\tau_{r}(\varphi)$ is invariant under an appropriate mapping of time reversal which interchanges past and future scattering data and reverses the direction of time. In Section 4.3, Theorem 4.6, we give a general existence criterion for the limit $\tau_{\Sigma}(\varphi):=\lim _{r \rightarrow+\infty} \tau_{r}(\varphi)$. For arbitrary dilated spatial regions symmetric with respect to the origin, $\tau_{\Sigma}(\varphi)$ is shown to be equal to the usual time delay plus a new contribution, which vanishes in the case of spherical spatial regions (see Remark 4.7). Transformations properties of $\tau_{\Sigma}(\varphi)$ under spatial translations are discussed in Remark 4.9 In the case of scattering by a short-ranged potential we derive stationary formulas for 
$\tau_{\Sigma}(\varphi)$ in Section 4.4 These results are also discussed in the context of classical scattering theory in Section 3 Section 2 contains some technical results on averaged characteristic functions.

\section{Averaged characteristic functions}

Let $\Sigma$ be a bounded open set in $\mathbb{R}^{d}$ containing 0 . For each $r>0$ we set $\Sigma_{r}:=\{r x \mid$ $x \in \Sigma$ \}. We shall simply say that $\Sigma$ is star-shaped (resp. symmetric) whenever $\Sigma$ is starshaped (resp. symmetric) with respect to 0 . Clearly $\Sigma$ is star-shaped iff $\Sigma_{r_{1}} \subset \Sigma_{r_{2}}$ for $0<r_{1} \leq r_{2}$. Moreover to each open star-shaped set $\Sigma$ we can associate a strictly positive continuous function $\ell_{\Sigma}$ on $\mathbb{S}^{d-1}$ defined by

$$
\ell_{\Sigma}(\omega):=\sup \{\mu \geq 0 \mid \mu \omega \in \Sigma\} .
$$

Conversely to each strictly positive continuous function $\ell$ on $\mathbb{S}^{d-1}$ one can associate a unique open star-shaped set $\Sigma$ such that $\ell=\ell_{\Sigma}$.

We shall also consider the following class of spatial regions $\Sigma\left(\mathbb{1}_{\Sigma}\right.$ stands for the characteristic function for $\Sigma$ ):

Assumption 2.1. $\Sigma$ is a bounded open set in $\mathbb{R}^{d}$ containing 0 and satisfying the condition

$$
\int_{0}^{+\infty} \mathrm{d} \mu\left[\mathbb{1}_{\Sigma}(\mu x)-\mathbb{1}_{\Sigma}(-\mu x)\right]=0, \quad \forall x \in \mathbb{R}^{d} .
$$

If $p \in \mathbb{R}^{d}$, then the number $\int_{0}^{+\infty} \mathrm{d} t \mathbb{1}_{\Sigma}(t p)$ is the sojourn time in $\Sigma$ of a free classical particle moving along the trajectory $t \mapsto x(t):=t p, t \geq 0$. Clearly if $\Sigma=-\Sigma$ (i.e. if $\Sigma$ is symmetric), then $\Sigma$ satisfies Assumption 2.1 Moreover if $\Sigma$ is star-shaped and satisfies Assumption 2.1 then $\Sigma=-\Sigma$.

Lemma 2.2. Let $\Sigma$ be a bounded open set in $\mathbb{R}^{d}$ containing 0 . Then:

(a) The limit

$$
R_{\Sigma}(x):=\lim _{\varepsilon \searrow 0}\left(\int_{\varepsilon}^{+\infty} \frac{\mathrm{d} \mu}{\mu} \mathbb{1}_{\Sigma}(\mu x)+\ln \varepsilon\right)
$$

exists for each $x \in \mathbb{R}^{d} \backslash\{0\}$.

(b) The (even) function $G_{\Sigma}: \mathbb{R}^{d} \backslash\{0\} \rightarrow \mathbb{R}$ given by

$$
G_{\Sigma}(x):=\frac{1}{2}\left[R_{\Sigma}(x)+R_{\Sigma}(-x)\right]
$$

satisfies

$$
G_{\Sigma}(x)=G_{\Sigma}\left(\frac{x}{|x|}\right)-\ln |x| .
$$

(c) If $\Sigma$ is star-shaped, then

$$
G_{\Sigma}(\omega)=\frac{1}{2}\left[\ln \left(\ell_{\Sigma}(\omega)\right)+\ln \left(\ell_{\Sigma}(-\omega)\right)\right]
$$

for each $\omega \in \mathbb{S}^{d-1}$. 
Proof. Let $x \in \mathbb{R}^{d} \backslash\{0\}$. Then point (a) follows from the equalities

$$
\begin{aligned}
\lim _{\varepsilon \searrow 0}\left(\int_{\varepsilon}^{+\infty} \frac{\mathrm{d} \mu}{\mu} \mathbb{1}_{\Sigma}(\mu x)+\ln \varepsilon\right) & =\int_{1}^{+\infty} \frac{\mathrm{d} \mu}{\mu} \mathbb{1}_{\Sigma}(\mu x)+\lim _{\varepsilon \searrow 0} \int_{\varepsilon}^{1} \frac{\mathrm{d} \mu}{\mu}\left[\mathbb{1}_{\Sigma}(\mu x)-1\right] \\
& =\int_{1}^{+\infty} \frac{\mathrm{d} \mu}{\mu} \mathbb{1}_{\Sigma}(\mu x)+\int_{0}^{1} \frac{\mathrm{d} \mu}{\mu}\left[\mathbb{1}_{\Sigma}(\mu x)-1\right] .
\end{aligned}
$$

Furthermore we have for $\lambda>0$

$$
\begin{aligned}
R_{\Sigma}(\lambda x) & =\lim _{\varepsilon \searrow 0}\left(\int_{\varepsilon}^{+\infty} \frac{\mathrm{d} \mu}{\mu} \mathbb{1}_{\Sigma}(\mu \lambda x)+\ln \varepsilon\right) \\
& =\lim _{\varepsilon \searrow 0}\left(\int_{\lambda \varepsilon}^{+\infty} \frac{\mathrm{d} \mu}{\mu} \mathbb{1}_{\Sigma}(\mu x)+\ln (\lambda \varepsilon)-\ln \lambda\right) \\
& =R_{\Sigma}(x)-\ln \lambda,
\end{aligned}
$$

which proves point (b). Finally point (c) follows from a direct computation.

We give now some properties of the functions $R_{\Sigma}$ and $G_{\Sigma}$, which follow easily from Lemma 2.2

Remark 2.3. (a) Let us consider $\left.\mathbb{R}_{+}^{*}:=\right] 0,+\infty[$ endowed with the multiplication as a Lie group with Haar measure $\frac{\mathrm{d} \mu}{\mu}$. Then $R_{\Sigma}$ is the (renormalized) average of $\mathbb{1}_{\Sigma}$ with respect to the action of $\mathbb{R}_{+}^{*}$ on $\mathbb{R}^{d}$.

(b) If $\Sigma$ is equal to the unit open ball $\mathcal{B}:=\left\{x \in \mathbb{R}^{d}|| x \mid<1\right\}$, then we have

$$
G_{\mathcal{B}}(x)=-\ln |x|, \quad x \in \mathbb{R}^{d} \backslash\{0\} .
$$

(c) To each set $\Sigma$ one can associate a unique symmetric star-shaped set $\widetilde{\Sigma}$ such that

$$
G_{\Sigma}=G_{\widetilde{\Sigma}}
$$

Indeed it suffices to take the symmetric star-shaped set $\widetilde{\Sigma}$ defined by the even, strictly positive, continuous function $\ell$ on $\mathbb{S}^{d-1}$ given by $\ell(\omega):=\exp \left(G_{\Sigma}(\omega)\right)$.

\section{Symmetrized time delay in classical scattering}

It is known [9. Sec. 2] that time delay in classical scattering (defined in terms of sojourn times) does exist only for sequences of dilated balls. In the sequel we recall the definition of the symmetrized time delay in classical scattering and show its existence for (more general) sequences of symmetric spatial regions (we are convinced that sequences $\Sigma_{r}$ with initial set $\Sigma$ satisfying Assumption 2.1 is the optimal case, but we prefer not to treat this case for the sake of simplicity). We also show that the symmetrized time delay and the usual time delay are equal for sequences of dilated balls. 
We adapt our approach from [9 Sec. 2]. In particular we suppose in the rest of the section that $\Sigma_{r}:=\{r x \mid x \in \Sigma\}$, where $\Sigma$ is a convex bounded open set (with smooth boundary) in $\mathbb{R}^{d}$ containing 0 . Let $V$ be a real $C^{2}$-potential with compact support. A scattering trajectory for $V$ is a map $\Phi: \mathbb{R} \ni t \mapsto(x(t), p(t)) \in \mathbb{R}^{d}$ solution of $\dot{x}(t)=p(t), \dot{p}(t)=-\nabla V(x(t))$ satisfying $|x(t)| \rightarrow \infty$ as $t \rightarrow \pm \infty$ and $E:=\frac{1}{2} p^{2}(t)+$ $V(x(t))>0$. A scattering trajectory has two asymptotic momenta $p_{ \pm}:=\lim _{t \rightarrow \pm \infty} p(t)$, where $\left|p_{ \pm}\right|=(2 E)^{1 / 2}=: p$ due to energy conservation. Let $\widetilde{x}(t):=x(t)-t p(t)$ and denote by $-t_{-}$and $t_{+}\left(t_{ \pm}>0\right)$ the times at which the particle enters and leaves the region $\Sigma_{r}$. If $r$ is large enough, then $\Sigma_{r}$ contains the support of $v$. Therefore $p\left( \pm t_{ \pm}\right)=p_{ \pm}$and

$$
t_{ \pm}=\mp \frac{p_{ \pm}}{p^{2}} \cdot\left(\widetilde{x}_{ \pm}-x_{ \pm}\right),
$$

where $\widetilde{x}_{ \pm}:=\widetilde{x}\left( \pm t_{ \pm}\right)$and $x_{ \pm}:=x\left( \pm t_{ \pm}\right)$. One can define three distinct sojourn times. The sojourn time in $\Sigma_{r}$ of the scattered particle is given by

$$
T_{r}:=t_{-}+t_{+}=\frac{1}{p^{2}}\left(p_{-} \cdot \widetilde{x}_{-}-p_{+} \cdot \widetilde{x}_{+}\right)-\frac{1}{p^{2}}\left(p_{-} \cdot x_{-}-p_{+} \cdot x_{+}\right) .
$$

The sojourn time in $\Sigma_{r}$ of the incoming free particle

$$
\left\{x^{0}(t), p^{0}(t)\right\}:=\left(x_{-}+p_{-}\left(t+t_{-}\right), p_{-}\right)
$$

with incoming momentum $p_{-}$, entering time $-t_{-}$and leaving time $t_{+}^{0}>0$ is

$$
T_{r}^{0}=t_{-}+t_{+}^{0}=\frac{1}{p^{2}}\left(p_{-} \cdot x_{+}^{0}-p_{-} \cdot x_{-}\right),
$$

where $x_{+}^{0}:=x^{0}\left(t_{+}^{0}\right)$. The sojourn time in $\Sigma_{r}$ of the outcoming free particle

$$
\left\{x^{0^{\prime}}(t), p^{0^{\prime}}(t)\right\}:=\left(x_{+}+p_{+}\left(t-t_{+}\right), p_{+}\right)
$$

with outcoming momentum $p_{+}$, leaving time $t_{+}$and entering time $-t_{-}^{0^{\prime}}<0$ is

$$
T_{r}^{0^{\prime}}=t_{-}^{0^{\prime}}+t_{+}=\frac{1}{p^{2}}\left(p_{+} \cdot x_{+}-p_{+} \cdot x_{-}^{0^{\prime}}\right),
$$

where $x_{-}^{0^{\prime}}:=x^{0^{\prime}}\left(-t_{-}^{0^{\prime}}\right)$. The (usual) time delay for the finite region $\Sigma_{r}$ is defined as

$$
\tau_{r}^{\text {in }}:=T_{r}-T_{r}^{0} .
$$

It is known that $\tau_{r}^{\text {in }}$ admits a limit as $r \rightarrow+\infty$ only if $\Sigma$ is a ball [9. Sec. 2]. In this case $\tau_{r}^{\text {in }}$ converges to the classical analogue $\tau^{\mathrm{cl}}$ of the Eisenbud-Wigner time delay [8]. On the other hand one can also define the symmetrized time delay:

$$
\tau_{r}:=T_{r}-\frac{1}{2}\left(T_{r}^{0}+T_{r}^{0^{\prime}}\right) .
$$


Remark 3.1. Let $\Phi: \mathbb{R} \ni t \mapsto(x(t), p(t)) \in \mathbb{R}^{d}$ be a scattering trajectory and $p_{ \pm} \equiv p_{ \pm}(\Phi), t_{ \pm} \equiv t_{ \pm}(\Phi), x_{ \pm} \equiv x_{ \pm}(\Phi), \widetilde{x}_{ \pm} \equiv \widetilde{x}_{ \pm}(\Phi)$ the associated scattering quantities. Consider the mapping f (of full time reversal) f : $\Phi \mapsto \Phi^{\mathrm{rev}}$, where $\Phi^{\mathrm{rev}}$ : $t \mapsto(x(-t),-p(-t))$. Then

$$
p_{ \pm} \circ \mathrm{f}=-p_{\mp}, \quad t_{ \pm} \circ \mathrm{f}=t_{\mp}, \quad x_{ \pm} \circ \mathrm{f}=x_{\mp}, \quad \widetilde{x}_{ \pm} \circ \mathrm{f}=\widetilde{x}_{\mp} .
$$

Furthermore, setting

$$
\tau_{r}^{\text {out }}:=\tau_{r}^{\text {in }} \circ \mathrm{f},
$$

we see that

$$
\tau_{r}=\frac{1}{2}\left(\tau_{r}^{\mathrm{in}}+\tau_{r}^{\mathrm{out}}\right) .
$$

Thus $\tau_{r}$ is the mean value of the usual time delay $\tau_{r}^{\text {in }}$ and of the time delay $\tau_{r}^{\text {out }}$ corresponding to the time reversed scattering process. In particular $\tau_{r}$ is invariant under full time reversal, namely one has $\tau_{r} \circ \mathrm{f}=\tau_{r}$ since $\mathrm{f}$ is an involution.

The symmetrized time delay (3.6) can be rewritten as

$$
\tau_{r}:=\tau_{r}^{(1)}+\tau_{r}^{(2)},
$$

where

$$
\begin{aligned}
\tau_{r}^{(1)} & :=\frac{1}{p^{2}}\left(p_{-} \cdot \widetilde{x}_{-}-p_{+} \cdot \widetilde{x}_{+}\right), \\
\tau_{r}^{(2)} & :=\frac{1}{2 p^{2}}\left(p_{+} \cdot x_{+}-p_{-} \cdot x_{-}-p_{-} \cdot x_{+}^{0}+p_{+} \cdot x_{-}^{0^{\prime}}\right) .
\end{aligned}
$$

Note that only free trajectories enter in the definition of $\tau_{r}^{(2)}$. As $r \rightarrow+\infty$ (i.e. as $t_{ \pm} \rightarrow$ $+\infty) \tau_{r}^{(1)}$ converges to $\tau^{\mathrm{cl}}$ [9. Sec. 2].

Let us now consider the convergence of $\tau_{r}^{(2)}$ as $r \rightarrow+\infty$. Let $y(t):=y_{0}+t p_{0}$ be an arbitrary free trajectory with $p_{0} \neq 0$. Let $y_{\mp}$ be the entrance and exit points of $y(t)$ in $\Sigma_{r}$. Since these points are independent of the parametrization we can assume that $y(t)=y_{0}+t \omega, \omega \in \mathbb{S}^{d-1}$. Thus

$$
r^{-1} y_{ \pm}\left(y_{0}, \omega, r\right)=y_{ \pm}\left(r^{-1} y_{0}, \omega, 1\right)
$$

and $y_{ \pm}(0, \omega, 1)= \pm \omega d( \pm \omega)$, where $d(\theta)$ is the distance from the origin to the boundary of $\Sigma$ in the direction $\theta \in \mathbb{S}^{d-1}$. Since $\Sigma$ is open the functions $y_{ \pm}$are continuous w.r.t. to $y_{0}$. Using [3.7) this implies that

$$
y_{ \pm}\left(y_{0}, \omega, r\right)= \pm r \omega d( \pm \omega)+o(r) .
$$

Applying $\left[3.8\right.$ to the two free trajectories $x^{0}(t)$ and $x^{0^{\prime}}(t)$, we get

$$
\begin{aligned}
& x_{-}=-r d\left(-\widehat{p}_{-}\right) \widehat{p}_{-}+o(r), \quad x_{+}^{0}=r d\left(\widehat{p}_{-}\right) \widehat{p}_{-}+o(r), \\
& x_{+}=r d\left(\widehat{p}_{+}\right) \widehat{p}_{+}+o(r), \quad x_{-}^{0^{\prime}}=-r d\left(-\widehat{p}_{+}\right) \widehat{p}_{+}+o(r),
\end{aligned}
$$


where $\widehat{p}_{ \pm}:=p_{ \pm} /\left|p_{ \pm}\right|$. If $\Sigma$ is a ball, then the remainder term in (3.8) is actually of order $O\left(r^{-1}\right)$, and

$$
x_{-}+x_{+}^{0}=O\left(r^{-1}\right), \quad x_{+}+x_{-}^{0^{\prime}}=O\left(r^{-1}\right) .
$$

It follows that

$$
\lim _{r \rightarrow+\infty} \tau_{r}^{(2)}=0 \quad \text { and } \quad \lim _{r \rightarrow+\infty} \tau_{r}=\lim _{r \rightarrow+\infty} \tau_{r}^{\text {in }}=\tau^{\mathrm{cl}} .
$$

Equations 3.11 show the identity of the usual time delay and of the symmetrized time delay in the case of spherical spatial regions.

For an arbitrary $\Sigma$, we get from (3.9)-(3.10):

$$
\tau_{r}^{(2)}=\frac{r}{2 p}\left[d\left(\widehat{p}_{+}\right)-d\left(-\widehat{p}_{+}\right)-d\left(\widehat{p}_{-}\right)+d\left(-\widehat{p}_{-}\right)\right]+o(r) .
$$

Clearly one has to impose that $d(\widehat{u})-d(-\widehat{u})=0$ for all $\widehat{u} \in \mathbb{S}^{d-1}$ in order to ensure the existence of the $\operatorname{limit}_{\lim } \rightarrow+\infty \tau_{r}^{(2)}$ for all possible scattering events. In consequence the limit of $\tau_{r}$ as $r \rightarrow+\infty$ does exist only if the set $\Sigma$ is symmetric.

\section{Symmetrized time delay in quantum scattering}

\subsection{Sojourn times}

In this section we recall some properties of the (quantum) sojourn times associated to the free Hamiltonian $H_{0}=-\frac{1}{2} \Delta$ and the full Hamitonian $H$ in $\mathcal{H}=\mathrm{L}^{2}\left(\mathbb{R}^{d}\right)$. We first recall some definitions.

$\Sigma$ is a bounded open set in $\mathbb{R}^{d}$ containing 0 , and $\Sigma_{r}=\{r x \mid x \in \Sigma\}$. For each $r \in \mathbb{R}^{*}$ we define the characteristic function

$$
\chi_{r}(x):=\mathbb{1}_{\Sigma}\left(\frac{x}{r}\right), \quad x \in \mathbb{R}^{d} .
$$

We write $\mathbb{1}_{H_{0}}(\cdot)$ for the spectral measure of $H_{0}$ and $Q$ for the (vector) position operator in $\mathcal{H}$. We set $\langle\cdot\rangle:=\sqrt{1+|\cdot|^{2}}$.

We will always assume that:

Assumption 4.1. The wave operators $W^{ \pm}$exist and are complete. The projections $\chi_{r}(Q)$ are locally $H$-smooth on $] 0,+\infty\left[\backslash \sigma_{\mathrm{pp}}(H)\right.$.

For latter use we introduce the following definition:

Definition 4.2. Let $s \geq 0$, then

$$
\mathcal{D}_{s}:=\left\{\varphi \in \mathcal{D}\left(\langle Q\rangle^{s}\right) \mid \mathbb{1}_{H_{0}}(J) \varphi=\varphi \text { for some compact set } J \text { in }\right] 0,+\infty\left[\backslash \sigma_{\mathrm{pp}}(H)\right\}
$$


It is clear that $\mathcal{D}_{s}$ is dense in $\mathcal{H}$ and that $\mathcal{D}_{s_{1}} \subset \mathcal{D}_{s_{2}}$ if $s_{1} \geq s_{2}$.

For $r>0$ and an appropriate scattering state $\varphi \in \mathrm{L}^{2}\left(\mathbb{R}^{d}\right)$, we define the free sojourn time

$$
T_{r}^{0}(\varphi):=\int_{-\infty}^{+\infty} \mathrm{d} t\left\|\chi_{r}(Q) \mathrm{e}^{-1 t H_{0}} \varphi\right\|^{2}
$$

and the full sojourn time

$$
T_{r}(\varphi):=\int_{-\infty}^{+\infty} \mathrm{d} t\left\|\chi_{r}(Q) \mathrm{e}^{-1 t H} W^{-} \varphi\right\|^{2} .
$$

Due to Assumption 4.1 one shows easily that these times are finite if $\varphi \in \mathcal{D}_{0}$. The time delay of the scattering process with incoming state $\varphi \in \mathcal{D}_{0}$ for $\Sigma_{r}$ is then defined as

$$
\tau_{r}^{\text {in }}(\varphi):=T_{r}(\varphi)-T_{r}^{0}(\varphi) .
$$

Since $S \mathcal{D}_{0} \subset \mathcal{D}_{0}$ one can also define the symmetrized time delay of the scattering process with incoming state $\varphi \in \mathcal{D}_{0}$ :

$$
\tau_{r}(\varphi):=T_{r}(\varphi)-\frac{1}{2}\left[T_{r}^{0}(\varphi)+T_{r}^{0}(S \varphi)\right] .
$$

Finally we define for each $r>0$ the auxiliary sojourn time $\tau_{r}^{\text {free }}(\varphi)$ (see [11 Sec. 2.1])

$$
\begin{aligned}
\tau_{r}^{\text {free }}(\varphi):=\frac{1}{2} & \int_{-\infty}^{0} \mathrm{~d} t\left(\left\|\chi_{r}(Q) \mathrm{e}^{-1 t H_{0}} \varphi\right\|^{2}-\left\|\chi_{r}(Q) \mathrm{e}^{-1 t H_{0}} S \varphi\right\|^{2}\right) \\
& +\frac{1}{2} \int_{0}^{+\infty} \mathrm{d} t\left(\left\|\chi_{r}(Q) \mathrm{e}^{-1 t H_{0}} S \varphi\right\|^{2}-\left\|\chi_{r}(Q) \mathrm{e}^{-1 t H_{0}} \varphi\right\|^{2}\right),
\end{aligned}
$$

which is also finite if $\varphi \in \mathcal{D}_{0}$.

Lemma 4.3. Suppose that Assumption 4.1] holds and let $\varphi \in \mathcal{D}_{0}$ be such that

$$
\left\|\left(W^{-}-\mathbb{1}\right) \mathrm{e}^{-1 t H_{0}} \varphi\right\| \in \mathrm{L}^{1}\left(\mathbb{R}_{-}, \mathrm{d} t\right)
$$

and

$$
\left\|\left(W^{+}-\mathbb{1}\right) \mathrm{e}^{-1 t H_{0}} S \varphi\right\| \in \mathrm{L}^{1}\left(\mathbb{R}_{+}, \mathrm{d} t\right) .
$$

Then

$$
\lim _{r \rightarrow+\infty}\left[\tau_{r}(\varphi)-\tau_{r}^{\text {free }}(\varphi)\right]=0
$$

Proof. For $t \in \mathbb{R}$, set

$$
f_{-}(t):=\left\|\mathrm{e}^{-1 t H} W^{-} \varphi-\mathrm{e}^{-1 t H_{0}} \varphi\right\| \quad \text { and } \quad f_{+}(t):=\left\|\mathrm{e}^{-1 t H} W^{+} \varphi-\mathrm{e}^{-1 t H_{0}} S \varphi\right\| .
$$

We know from Hypotheses (4.13) and (4.14) that $f_{ \pm} \in \mathrm{L}^{1}\left(\mathbb{R}_{ \pm}\right)$. Using the inequality

$$
\left|\|u\|^{2}-\|v\|^{2}\right| \leq\|u-v\|(\|u\|+\|v\|), \quad u, v \in \mathcal{H},
$$


we obtain the estimates

$$
\begin{aligned}
\left|\left\|\chi_{r}(Q) \mathrm{e}^{-1 t H} W^{-} \varphi\right\|^{2}-\left\|\chi_{r}(Q) \mathrm{e}^{-1 t H_{0}} \varphi\right\|^{2}\right| & \leq 2 f_{-}(t)\|\varphi\|, \\
\left|\left\|\chi_{r}(Q) \mathrm{e}^{-1 t H} W^{-} \varphi\right\|^{2}-\left\|\chi_{r}(Q) \mathrm{e}^{-1 t H_{0}} S \varphi\right\|^{2}\right| & \leq 2 f_{+}(t)\|\varphi\| .
\end{aligned}
$$

Since $\mathrm{s}-\lim _{r \rightarrow+\infty} \chi_{r}(Q)=1$, then the scalars on the 1.h.s. above converge to 0 as $r \rightarrow+\infty$. Thus the claim follows from 4.12 and Lebesgue's dominated convergence theorem.

\subsection{Time reversal}

We now collect some elementary remarks related to time reversal for the (complete) scattering system $\left\{H_{0}, H\right\}$.

Time reversal is implemented by the antiunitary involution

$$
\mathcal{H} \ni \varphi \mapsto \bar{\varphi} \text {. }
$$

The Hamiltonian $H$ is invariant under time reversal if

$$
H \bar{\varphi}=\overline{H \varphi}, \quad \varphi \in \mathcal{D}(H) .
$$

In such a case one has the identities $\mathrm{e}^{-1 t H} \bar{\varphi}=\overline{\mathrm{e}^{t t H} \varphi}, W^{ \pm} \bar{\varphi}=\overline{W^{\mp} \varphi}$ and

$$
S \bar{\varphi}=\overline{S^{-1} \varphi}
$$

for each $\varphi \in \mathcal{H}$. Consider the bijection

$$
\mathrm{f}: \mathcal{H} \rightarrow \mathcal{H}, \quad \varphi \mapsto \overline{S \varphi}
$$

which we call full time reversal. The map $\mathrm{f}$ corresponds to time reversal for the full scattering process, i.e. it interchanges past and future scattering data and reverses the direction of time. Furthermore one sees easily from (4.16) that $\mathrm{f}$ is an antiunitary involution.

In order to give a rigourous interpretation of full time reversal we introduce the space $\mathcal{E}$ of scattering trajectories, i.e. the space of continuous maps

$$
\mathbb{R} \ni t \mapsto \Phi(t) \in \mathcal{H},
$$

such that

$$
{ }_{1}\left(\partial_{t} \Phi\right)(t)=H \Phi(t) \forall t \in \mathbb{R} \text { (in the weak sense) and } \quad \mathrm{w}-\lim _{t \rightarrow \pm \infty} \Phi(t)=0 .
$$

The space $\mathcal{E}$ is invariant under the involution

$$
R: \mathcal{E} \rightarrow \mathcal{E}, \quad(R \Phi)(t):=\overline{\Phi(-t)} .
$$

One can associate to a trajectory $\Phi \in \mathcal{E}$ a vector $\varphi:=T(\Phi) \in \mathcal{H}$ defined by the constraint

$$
\mathrm{s}-\lim _{t \rightarrow-\infty}\left(\Phi(t)-\mathrm{e}^{-1 t H_{0}} \varphi\right)=0 .
$$


Due to the completeness of the wave operators we know that $T: \mathcal{E} \rightarrow \mathcal{H}$ is bijective, and we have

$$
\mathrm{f}(\varphi)=\left(T R T^{-1}\right)(\varphi), \quad \varphi \in \mathcal{H} .
$$

Equation (4.17) provides a rigourous meaning to full time reversal as a map interchanging past and future scattering data and reversing the direction of time.

Lemma 4.4. Assume that $H$ is invariant under time reversal, and set

$$
\tau_{r}^{\text {out }}(\varphi):=\left(\tau_{r}^{\text {in }} \circ \mathrm{f}\right)(\varphi)
$$

Then one has the equalities

$$
\tau_{r}(\varphi)=\frac{1}{2}\left[\tau_{r}^{\text {in }}(\varphi)+\tau_{r}^{\text {out }}(\varphi)\right] \text { and } \tau_{r}(\varphi)=\left(\tau_{r} \circ \mathrm{f}\right)(\varphi) .
$$

Thus $\tau_{r}(\varphi)$ is the mean value of the usual time delay $\tau_{r}^{\text {in }}(\varphi)$ and of the time delay $\tau_{r}^{\text {out }}(\varphi)$ corresponding to the time reversed scattering process. In particular $\tau_{r}(\varphi)$ is invariant under full time reversal.

Proof. Since $H_{0}$ is invariant under time reversal, one gets

$$
T_{r}^{0}(\varphi)=T_{r}^{0}(\bar{\varphi}) .
$$

This together with time reversal invariance of $H$ yields

$$
T_{r}(\overline{S \varphi})=T_{r}(\varphi)
$$

Thus

$$
\tau_{r}^{\text {out }}(\varphi)=\tau_{r}^{\text {in }}(\overline{S \varphi})=T_{r}(\overline{S \varphi})-T_{r}^{0}(\overline{S \varphi})=T_{r}(\varphi)+T_{r}^{0}(S \varphi),
$$

which implies the first identity in (4.18). The second identity follows from the fact that $\mathrm{f}$ is an involution.

\subsection{Time delay}

In the present section we shall give the proof of the existence of the symmetrized time delay. We first fix some notation. If $A, B$ are two symmetric operators, then we set for each $\varphi \in \mathcal{D}(A) \cap \mathcal{D}(B)$ :

$$
(\varphi,[A, B] \varphi):=(A \varphi, B \varphi)-(B \varphi, A \varphi) .
$$

If $q$ is a quadratic form with domain $\mathcal{D}(q)$, and $S$ is unitary, then we set for each $\varphi \in$ $\mathcal{D}(q) \cap S^{-1} \mathcal{D}(q)$ :

$$
\left(\varphi, S^{*}[q, S] \varphi\right):=q(S \varphi)-q(\varphi) .
$$

If $A$ is an operator with domain $\mathcal{D}(A)$ and $S$ is unitary, then we define the operator $S^{*}[A, S]$ with domain $\mathcal{D}(A) \cap S^{-1} \mathcal{D}(A)$ by

$$
S^{*}[A, S]:=S^{*} A S-A .
$$

We also recall that the function $G_{\Sigma}$ was introduced in Section 2 and that $\mathcal{D}_{2} \subset \mathcal{D}\left(Q^{2}\right) \cap$ $\mathcal{D}\left(G_{\Sigma}(P)\right)$. Therefore the quadratic form $1\left[Q^{2}, G_{\Sigma}(P)\right]$ is well defined on $\mathcal{D}_{2}$.

The proof of the next proposition can be found in the appendix. 
Proposition 4.5. Let $\Sigma$ satisfy Assumption 2.1] Suppose that Assumption 4.1 is verified. Then we have for all $\varphi \in \mathcal{D}_{2}$ the equality

$$
\begin{aligned}
& \lim _{r \rightarrow+\infty} \int_{0}^{+\infty} \mathrm{d} t\left(\varphi,\left(\mathrm{e}^{1 t P^{2} / 2} \chi_{r}(Q) \mathrm{e}^{-1 t P^{2} / 2}-\mathrm{e}^{-1 t P^{2} / 2} \chi_{r}(Q) \mathrm{e}^{1 t P^{2} / 2}\right) \varphi\right) \\
& =-\left(\varphi, 1\left[Q^{2}, G_{\Sigma}(P)\right] \varphi\right) .
\end{aligned}
$$

We are now in a position to give the proof of our main theorem. It involves the operator

$$
A_{0}:=\frac{1}{2}\left(\frac{P}{P^{2}} \cdot Q+Q \cdot \frac{P}{P^{2}}\right),
$$

which is well-defined and symmetric on $\mathcal{D}_{1}$.

Theorem 4.6. Let $\Sigma$ satisfy Assumption 2.1 Suppose that Assumption 4.1 is verified. Let $\varphi \in \mathcal{D}_{2}$ satisfy 44.13, 44.14 and $S \varphi \in \mathcal{D}_{2}$. Then the limit $\tau_{\Sigma}(\varphi)=\lim _{r \rightarrow+\infty} \tau_{r}(\varphi)$ exists, and one has

$$
\tau_{\Sigma}(\varphi)=-\frac{1}{2}\left(\varphi, S^{*}\left[1\left[Q^{2}, G_{\Sigma}\left(\frac{P}{|P|}\right)\right], S\right] \varphi\right)-\left(\varphi, S^{*}\left[A_{0}, S\right] \varphi\right) .
$$

The quadratic form $1\left[Q^{2}, G_{\Sigma}\left(\frac{P}{|P|}\right)\right]$ and the operator $A_{0}$ are well defined on $\mathcal{D}_{2}$, so all the commutators in the above formula are well defined since $\varphi, S \varphi \in \mathcal{D}_{2}$.

Proof. The expression (4.12) for $\tau_{r}^{\text {free }}(\varphi)$ can be rewritten as

$$
\begin{aligned}
\tau_{r}^{\text {free }}(\varphi)= & -\frac{1}{2} \int_{0}^{+\infty} \mathrm{d} t\left(\varphi,\left(\mathrm{e}^{1 t P^{2} / 2} \chi_{r}(Q) \mathrm{e}^{-1 t P^{2} / 2}-\mathrm{e}^{-1 t P^{2} / 2} \chi_{r}(Q) \mathrm{e}^{1 t P^{2} / 2}\right) \varphi\right) \\
& +\frac{1}{2} \int_{0}^{+\infty} \mathrm{d} t\left(S \varphi,\left(\mathrm{e}^{1 t P^{2} / 2} \chi_{r}(Q) \mathrm{e}^{-1 t P^{2} / 2}-\mathrm{e}^{-1 t P^{2} / 2} \chi_{r}(Q) \mathrm{e}^{1 t P^{2} / 2}\right) S \varphi\right) .
\end{aligned}
$$

Applying Proposition 4.5, we get

$$
\begin{aligned}
\lim _{r \rightarrow+\infty} \tau_{r}^{\text {free }}(\varphi) & =\frac{1}{2}\left(\varphi, 1\left[Q^{2}, G_{\Sigma}(P)\right] \varphi\right)-\frac{1}{2}\left(S \varphi, 1\left[Q^{2}, G_{\Sigma}(P)\right] S \varphi\right) \\
& =-\frac{1}{2}\left(\varphi, S^{*}\left[1\left[Q^{2}, G_{\Sigma}(P)\right], S\right] \varphi\right) .
\end{aligned}
$$

By Lemma2.2(b), we have $G_{\Sigma}(P)=G_{\Sigma}\left(\frac{P}{|P|}\right)-\ln |P|$, and we know from [2], Sec. 2] that

$$
\frac{1}{2}\left[Q^{2},-\ln |P|\right]=A_{0},
$$

as quadratic forms on $\mathcal{D}_{2}$. This yields

$$
\lim _{r \rightarrow+\infty} \tau_{r}^{\mathrm{free}}(\varphi)=-\frac{1}{2}\left(\varphi, S^{*}\left[1\left[Q^{2}, G_{\Sigma}\left(\frac{P}{|P|}\right)\right], S\right] \varphi\right)-\left(\varphi, S^{*}\left[A_{0}, S\right] \varphi\right) .
$$

We conclude by using Lemma 4.3 
Remark 4.7. The second term in Formula (4.20) coincides with the usual value of time delay; it is equal to the limit (for $\Sigma$ spherical) of $\tau_{r}^{\text {in }}(\varphi)$ as $r \rightarrow+\infty$ (see [2] Prop. 1]). The first term is a new contribution to time delay determined by the shape of the set $\Sigma$. If $\Sigma$ is spherical, this contribution vanishes due to Remark 2.3 (b), and then one gets (under the hypotheses of Theorem 4.6 the equality

$$
\lim _{r \rightarrow+\infty} \tau_{r}(\varphi)=\lim _{r \rightarrow+\infty} \tau_{r}^{\text {in }}(\varphi) .
$$

Remark 4.8. Under the hypotheses of Theorem 4.6 the two following facts are true whenever $\Sigma$ is an open bounded set containing the origin (see [9] Sec. 3]):

(a) The equality $\lim _{r \rightarrow+\infty}\left[\tau_{r}(\varphi)-\tau_{r}^{\text {free }}(\varphi)\right]=0$ holds.

(b) The difference

$$
\begin{aligned}
& \int_{0}^{+\infty} \mathrm{d} t\left(S \varphi,\left[\mathrm{e}^{i t P^{2}} \chi_{r}(Q) \mathrm{e}^{-i t P^{2}}-\mathrm{e}^{-i t P^{2}} \chi_{r}(Q) \mathrm{e}^{i t P^{2}}, S\right] \varphi\right) \\
& -r \int_{0}^{+\infty} \mathrm{d} u\left(S \varphi,\left[\left|H_{0}\right|^{-1 / 2}\left(\mathbb{1}_{\Sigma}\left(u \frac{P}{|P|}\right)-\mathbb{1}_{\Sigma}\left(-u \frac{P}{|P|}\right)\right), S\right] \varphi\right)
\end{aligned}
$$

remains bounded as $r \rightarrow \infty$.

The integrand in the second term in (b) can be written as

$$
\left(S \varphi,\left|H_{0}\right|^{-1 / 2}[M(P), S] \varphi\right),
$$

where

$$
M(x)=|x| \int_{0}^{+\infty} \mathrm{d} \mu\left[\mathbb{1}_{\Sigma}(\mu x)-\mathbb{1}_{\Sigma}(-\mu x)\right], \quad x \in \mathbb{R}^{d} .
$$

The combination of facts (a) and (b) shows that $\tau_{r}(\varphi)$ can have a limit for $\varphi$ in a dense set $\mathcal{E} \subset \mathcal{H}$ only if

$$
\left(S \varphi,\left|H_{0}\right|^{-1 / 2}[M(P), S] \varphi\right)=0, \quad \forall \varphi \in \mathcal{E},
$$

which implies that $[M(P), S]=0$. Therefore, if the scattering operator $S$ has no other symmetry than $\left[S, P^{2}\right]=0$, one has $M(P)=F\left(P^{2}\right)$ for some function $F$, and it follows that $M \equiv 0$ since $M(x)=-M(-x)$. In consequence $\tau_{r}(\varphi)$ can have a limit for $\varphi$ in a dense set $\mathcal{E}$ only if $\Sigma$ satisfies Assumption 2.1

Remark 4.9. One could also consider time delay for sets $\Sigma_{r}$ translated by a vector a $\in$ $\mathbb{R}^{d}$. Obviously this is equivalent to determining the time delay 4.20 when the origin of the spatial coordinate system is translated to the point a. In this case one has

$$
\begin{aligned}
& \varphi \mapsto \varphi_{a}:=\mathrm{e}^{1 P \cdot a} \varphi, \\
& S \mapsto S_{a}:=\mathrm{e}^{1 P \cdot a} S \mathrm{e}^{-1 P \cdot a},
\end{aligned}
$$


and $\tau_{\Sigma}(\varphi)$ becomes

$$
\tau_{\Sigma}^{a}(\varphi):=-\frac{1}{2}\left(\varphi_{a}, S_{a}^{*}\left[1\left[Q^{2}, G_{\Sigma}\left(\frac{P}{|P|}\right)\right], S_{a}\right] \varphi_{a}\right)-\left(\varphi_{a}, S_{a}^{*}\left[A_{0}, S_{a}\right] \varphi_{a}\right) .
$$

Using the formulas

$$
\begin{aligned}
\mathrm{e}^{-1 P \cdot a}\left[Q^{2}, G_{\Sigma}\left(\frac{P}{|P|}\right)\right] \mathrm{e}^{1 P \cdot a} & =\left[Q^{2}, G_{\Sigma}\left(\frac{P}{|P|}\right)\right]-2 a \cdot\left[Q, G_{\Sigma}\left(\frac{P}{|P|}\right)\right] \\
\mathrm{e}^{-1 P \cdot a} A_{0} \mathrm{e}^{1 P \cdot a} & =A_{0}-a \cdot \frac{P}{P^{2}}
\end{aligned}
$$

one gets

$$
\tau_{\Sigma}^{a}(\varphi)=\tau_{\Sigma}(\varphi)+a \cdot\left(\varphi, S^{*}\left[1\left[Q, G_{\Sigma}\left(\frac{P}{|P|}\right)\right], S\right] \varphi\right)+a \cdot\left(\varphi, S^{*}\left[\frac{P}{P^{2}}, S\right] \varphi\right) .
$$

Due to its very definition time delay given by Formula 4.21 is clearly covariant under spatial translations.

\subsection{Stationary formulas}

In the sequel we suppose that $\Sigma$ satisfy Assumption 2.1 We know from Remark 2.3 (c) that there exists a symmetric star-shaped set $\widetilde{\Sigma}$ such that

$$
\tau^{\Sigma}(\varphi)=\tau^{\widetilde{\Sigma}}(\varphi)
$$

for $\varphi$ satisfying the hypotheses of Theorem 4.6 Thus with no loss of generality we may assume that $\Sigma$ is symmetric and star-shaped. We also assume that the boundary $\partial \Sigma$ of $\Sigma$ is a $C^{2}$ hypersurface, so that the functions $\ell_{\Sigma}$ and $G_{\Sigma}$ (see Formulas (2.3) and (2.4) associated to $\Sigma$ are $C^{2}$. In such a case one has

$$
\frac{1}{2}\left[Q^{2}, G_{\Sigma}\left(\frac{P}{|P|}\right)\right]=-\frac{1}{2}\left[Q \cdot \nabla G_{\Sigma}\left(\frac{P}{|P|}\right)+\nabla G_{\Sigma}\left(\frac{P}{|P|}\right) \cdot Q\right]=: B_{\Sigma},
$$

as quadratic forms on $\mathcal{D}_{2}$ (note that $B_{\Sigma}$ is a well-defined symmetric operator on $\mathcal{D}_{1}$ ). Thus we can rewrite $\tau^{\Sigma}(\varphi)$ as

$$
\tau_{\Sigma}(\varphi)=-\left(\varphi, S^{*}\left[B_{\Sigma}, S\right] \varphi\right)-\left(\varphi, S^{*}\left[A_{0}, S\right] \varphi\right)
$$

Let $\mathscr{U}: \mathrm{L}^{2}\left(\mathbb{R}^{d}\right) \rightarrow \int_{\mathbb{R}_{+}}^{\oplus} \mathrm{d} \lambda \mathrm{L}^{2}\left(\mathbb{S}^{d-1}\right)$ be the spectral transformation for $H_{0}$, i.e. the unitary mapping defined by

$$
(\mathscr{U} \varphi)(\lambda, \omega)=(2 \lambda)^{(d-2) / 4}(\mathscr{F} \varphi)(\sqrt{2 \lambda} \omega)
$$

where $\mathscr{F}$ denotes the Fourier transform. One has

$$
\mathscr{U} H_{0} \mathscr{U}^{-1}=\int_{\mathbb{R}_{+}}^{\oplus} \mathrm{d} \lambda \lambda \text { and } \mathscr{U} S \mathscr{U}^{-1}=\int_{\mathbb{R}_{+}}^{\oplus} \mathrm{d} \lambda S(\lambda),
$$

where $\{S(\lambda)\}_{\lambda \geq 0} \subset \mathscr{B}\left(\mathrm{L}^{2}\left(\mathbb{S}^{d-1}\right)\right)$ is the scattering matrix for the pair $\left\{H_{0}, H\right\}$. For shortness we shall set $\varphi(\lambda):=(T \varphi)(\lambda, \cdot) \in \mathrm{L}^{2}\left(\mathbb{S}^{d-1}\right)$. 
If the interaction $V:=H-H_{0}$ is a potential sufficiently short-ranged, then there exists a dense set $\mathcal{E} \subset \mathcal{H}$ such that the hypotheses of Theorem 4.6 are satisfied for any $\varphi \in \mathcal{E}$ (a precise definition of $V$ and $\mathcal{E}$ can be found in [2] Prop.2]). Furthermore the function $\lambda \mapsto S(\lambda)$ is strongly continuously differentiable on $\mathcal{E}$, and the second term in 4.22 is equal to the Eisenbud-Wigner time delay for any $\varphi \in \mathcal{E}$ :

$$
-\left(\varphi, S^{*}\left[A_{0}, S\right] \varphi\right)=-1 \int_{0}^{\infty} \mathrm{d} \lambda\left(\varphi(\lambda), S(\lambda)^{*}\left(\frac{\mathrm{d} S(\lambda)}{\mathrm{d} \lambda}\right) \varphi(\lambda)\right)_{\mathrm{L}^{2}\left(\mathbb{S}^{d-1}\right)} \equiv\left(\varphi, \tau_{\mathrm{E}-\mathrm{w}} \varphi\right) .
$$

Let us now consider the first term in 4.22). Since the function $x \mapsto G_{\Sigma}\left(\frac{x}{|x|}\right)$ is homogeneous of degree 0 , one has

$$
x \cdot\left(\nabla G_{\Sigma}\right)\left(\frac{x}{|x|}\right)=0,
$$

namely the vector field $\left(\nabla G_{\Sigma}\right)\left(\frac{x}{|x|}\right)$ is orthogonal to the radial direction. In fact a direct calculation shows that

$$
\mathscr{U} B_{\Sigma} \mathscr{U}^{-1}=\int_{\mathbb{R}_{+}}^{\oplus} \mathrm{d} \lambda \lambda^{-1} b_{\Sigma}\left(\omega, \partial_{\omega}\right),
$$

where $b_{\Sigma}\left(\omega, \partial_{\omega}\right)$ is a symmetric first order differential operator on $\mathbb{S}^{d-1}$ with $C^{1}$ coefficients. Therefore the operator $\mathscr{U} B_{\Sigma} \mathscr{U}^{-1}$ is essentially selfadjoint on $\mathscr{U} \mathcal{D}_{1}$, and its closure is decomposable in the spectral representation of $H_{0}$, i.e.

$$
\overline{\mathscr{U} B_{\Sigma} \mathscr{U}^{-1}}=\mathscr{U} \overline{B_{\Sigma}} \mathscr{U}^{-1} \equiv \int_{\mathbb{R}_{+}}^{\oplus} \mathrm{d} \lambda B_{\Sigma}(\lambda) .
$$

This yields the equality

$$
\begin{aligned}
\tau_{\Sigma}(\varphi)=-\int_{0}^{+\infty} & \mathrm{d} \lambda\left(\varphi(\lambda), S^{*}(\lambda)\left[B_{\Sigma}(\lambda), S(\lambda)\right] \varphi(\lambda)\right)_{\mathrm{L}^{2}\left(\mathbb{S}^{d-1}\right)} \\
& -1 \int_{0}^{+\infty} \mathrm{d} \lambda\left(\varphi(\lambda), S(\lambda)^{*}\left(\frac{\mathrm{d} S(\lambda)}{\mathrm{d} \lambda}\right) \varphi(\lambda)\right)_{\mathrm{L}^{2}\left(\mathbb{S}^{d-1}\right)} .
\end{aligned}
$$

In consequence the time delay 4.22 is the sum of two contributions, each of these being the expectation value of an operator decomposable in the spectral representation of $H_{0}$.

\section{Appendix}

Proof of Proposition 4.5 (i) For any $F \in \mathrm{L}^{\infty}\left(\mathbb{R}^{d}\right)$ and $s \in \mathbb{R}$ one has

$$
\begin{aligned}
& \mathrm{e}^{1 s P^{2} / 2} F(Q) \mathrm{e}^{-1 s P^{2} / 2}=F(Q+s P), \\
& \mathrm{e}^{-1 s Q^{2} / 2} F(P) \mathrm{e}^{1 s Q^{2} / 2}=F(P+s Q),
\end{aligned}
$$

which imply the identity

$$
\mathrm{e}^{1 t P^{2} / 2} F(Q) \mathrm{e}^{-1 t P^{2} / 2}=Z_{-1 / t} F(t P) Z_{1 / t},
$$


where $t \in \mathbb{R}^{*}$ and $Z_{\tau}:=\mathrm{e}^{\mathrm{i} \tau Q^{2} / 2}$. Formula (4.24) and the change of variables $\mu=r t^{-1}$, $\nu=r^{-1}$, lead to the equalities

$$
\begin{aligned}
& \int_{0}^{+\infty} \mathrm{d} t\left(\varphi,\left(\mathrm{e}^{1 t P^{2} / 2} \chi_{r}(Q) \mathrm{e}^{-1 t P^{2} / 2}-\mathrm{e}^{-1 t P^{2} / 2} \chi_{r}(Q) \mathrm{e}^{1 t P^{2} / 2}\right) \varphi\right) \\
& =\int_{0}^{+\infty} \frac{\mathrm{d} \mu}{\nu \mu^{2}}\left(\varphi,\left(Z_{-\nu \mu} \chi_{\mu}(P) Z_{\nu \mu}-Z_{\nu \mu} \chi_{-\mu}(P) Z_{-\nu \mu}\right) \varphi\right) .
\end{aligned}
$$

One has also

$$
\int_{0}^{+\infty} \frac{\mathrm{d} \mu}{\mu^{2}}\left[\chi_{\mu}(P)-\chi_{-\mu}(P)\right]=\int_{0}^{+\infty} \mathrm{d} s[\chi(s P)-\chi(-s P)]=0
$$

due to Assumption 2.1 Hence the 1.h.s of 4.19) can be written as

$$
K_{\infty}(\varphi):=\lim _{\nu \searrow 0} \int_{0}^{+\infty} \mathrm{d} \mu K_{\nu, \mu}(\varphi)
$$

where

$$
\begin{aligned}
K_{\nu, \mu}(\varphi):=\frac{1}{\nu \mu^{2}} & \left(\varphi,\left[Z_{-\nu \mu} \chi_{\mu}(P) Z_{\nu \mu}-\chi_{\mu}(P)\right] \varphi\right) \\
& -\frac{1}{\nu \mu^{2}}\left(\varphi,\left[Z_{\nu \mu} \chi_{-\mu}(P) Z_{-\nu \mu}-\chi_{-\mu}(P)\right] \varphi\right) .
\end{aligned}
$$

(ii) To prove the statement, we shall show that one may interchange the limit and the integral in 4.25, by invoking Lebesgue's dominated convergence theorem. This will be done in (iii) below. If one assumes that this interchange is justified for the moment, then direct calculations give

$$
\begin{aligned}
K_{\infty}(\varphi) & =\left.\int_{0}^{+\infty} \mathrm{d} \mu \frac{\mathrm{d}}{\mathrm{d} \nu} K_{\nu, \mu}(\varphi)\right|_{\nu=0} \\
& =-\frac{1}{2} \int_{0}^{+\infty} \frac{\mathrm{d} \mu}{\mu}\left(\varphi, 1\left(\left[Q^{2}, \chi_{\mu}(P)\right]+\left[Q^{2}, \chi_{-\mu}(P)\right]\right) \varphi\right) .
\end{aligned}
$$

Due to Lemma2.2 (a) we have

$$
\begin{aligned}
& \int_{0}^{+\infty} \frac{\mathrm{d} \mu}{\mu}\left(\varphi,\left[Q^{2}, \chi_{\mu}(P)\right] \varphi\right) \\
& =\lim _{\varepsilon \searrow 0} \int_{\varepsilon}^{+\infty} \frac{\mathrm{d} \mu}{\mu}\left[\left(Q^{2} \varphi, \chi_{\mu}(P) \varphi\right)-\left(\chi_{\mu}(P) \varphi, Q^{2} \varphi\right)\right] \\
& =\lim _{\varepsilon \searrow 0}\left[\left(Q^{2} \varphi,\left(\int_{\varepsilon}^{+\infty} \frac{\mathrm{d} \mu}{\mu} \chi_{\mu}(P)+\ln \varepsilon\right) \varphi\right)-\left(\left(\int_{\varepsilon}^{+\infty} \frac{\mathrm{d} \mu}{\mu} \chi_{\mu}(P)+\ln \varepsilon\right) \varphi, Q^{2} \varphi\right)\right] \\
& =\left(\varphi,\left[Q^{2}, R_{\Sigma}(P)\right] \varphi\right) .
\end{aligned}
$$

This together with (4.26) lead to the desired equality, that is

$$
K_{\infty}(\varphi)=-\frac{1}{2}\left(\varphi, 1\left[Q^{2}, R_{\Sigma}(P)+R_{\Sigma}(-P)\right] \varphi\right)=-\left(\varphi, 1\left[Q^{2}, G_{\Sigma}(P)\right] \varphi\right) .
$$


(iii) To apply Lebesgue's dominated convergence theorem to 4.25 we need to bound $K_{\nu, \mu}(\varphi)$ uniformly in $\nu$ by a function in $\mathrm{L}^{1}\left(\mathbb{R}_{+}, \mathrm{d} \mu\right)$. We do this separately for $\mu \leq 1$ and for $\mu \geq 1$.

We begin with the case $\mu \leq 1$. Write $K_{\nu, \mu}(\varphi)$ as

$$
K_{\nu, \mu}(\varphi)=F_{\nu, \mu}(\varphi)-F_{\nu,-\mu}(\varphi)
$$

where

$$
\begin{aligned}
F_{\nu, \mu}(\varphi) & =\frac{1}{\nu \mu^{2}}\left[\left(Z_{\nu \mu} \varphi, \chi_{\mu}(P) Z_{\nu \mu} \varphi\right)-\left(\varphi, \chi_{\mu}(P) \varphi\right)\right] \\
& =\frac{1}{\mu}\left(\left(\frac{Z_{\nu \mu}-1}{\nu \mu}\right) \varphi, \chi_{\mu}(P) Z_{\nu \mu} \varphi\right)+\frac{1}{\mu}\left(\chi_{\mu}(P) \varphi,\left(\frac{Z_{\nu \mu}-1}{\nu \mu}\right) \varphi\right) .
\end{aligned}
$$

Due to the spectral theorem, we have

$$
\left\|\left(\frac{Z_{ \pm \nu \mu}-1}{\nu \mu}\right) \varphi\right\| \leq \text { Const. }\left\|\langle Q\rangle^{2} \varphi\right\| .
$$

Let $0<\ell<\frac{1}{2}$, then $|P|^{-\ell}\langle Q\rangle^{-2}$ is bounded (after conjugation by a Fourier transform this follows from the fact that $|Q|^{-\ell}$ is $P^{2}$-bounded [1 Prop. 2.28]). Since $\Sigma$ is bounded, we have

$$
\left|\mu^{-1} \xi\right|^{\ell}\left|\chi_{ \pm \mu}(\xi)\right| \leq \text { Const. }
$$

Therefore

$$
\begin{aligned}
\mu^{-1}\left\|\chi_{ \pm \mu}(P) \varphi\right\| & =\mu^{\ell-1}\left\|\left|\mu^{-1} P\right|^{\ell} \chi_{ \pm \mu}(P)|P|^{-\ell}\langle Q\rangle^{-2}\langle Q\rangle^{2} \varphi\right\| \\
& \leq \text { Const. } \mu^{\ell-1}\left\|\langle Q\rangle^{2} \varphi\right\|,
\end{aligned}
$$

and

$$
\mu^{-1}\left\|\chi_{ \pm \mu}(P) Z_{ \pm \nu \mu} \varphi\right\| \leq \text { Const. } \mu^{\ell-1}\left\|\langle Q\rangle^{2} \varphi\right\| .
$$

From 4.27, 4.28 and 4.29) we get the estimates

$$
\left|F_{\nu, \pm \mu}(\varphi)\right| \leq \text { Const. } \mu^{\ell-1}\left\|\langle Q\rangle^{2} \varphi\right\|^{2}
$$

Thus we have

$$
\left|K_{\nu, \mu}(\varphi)\right| \leq \text { Const. } \mu^{\ell-1}\left\|\langle Q\rangle^{2} \varphi\right\|^{2},
$$

which shows that $K_{\nu, \mu}(\varphi)$ is bounded uniformly in $\nu$ by a function in $\mathrm{L}^{1}([0,1], \mathrm{d} \mu)$.

We consider now the case $\mu \geq 1$. Since $\varphi=\mathbb{1}_{J}\left(H_{0}\right) \varphi$ for some compact set $J$, there exists $\mu_{0} \geq 0$ such that

$$
\left(\varphi, \chi_{\mu}(P) \varphi\right)=\left(\varphi, \chi_{-\mu}(P) \varphi\right)=(\varphi, \varphi), \quad \forall \mu \geq \mu_{0} .
$$

Hence for $\mu \geq \mu_{0}$, we have

$$
\begin{aligned}
\left|K_{\nu, \mu}(\varphi)\right| & =\frac{1}{\nu \mu^{2}}\left|\left(\varphi, Z_{-\nu \mu} \chi_{\mu}(P) Z_{\nu \mu} \varphi\right)-\left(\varphi, Z_{\nu \mu} \chi_{-\mu}(P) Z_{-\nu \mu} \varphi\right)\right| \\
& =\frac{1}{\nu \mu^{2}}\left|\left(\left\|\chi_{\mu}(P) Z_{\nu \mu} \varphi\right\|^{2}-\left\|Z_{\nu \mu} \varphi\right\|^{2}\right)-\left(\left\|\chi_{-\mu}(P) Z_{-\nu \mu} \varphi\right\|^{2}-\left\|Z_{-\nu \mu} \varphi\right\|^{2}\right)\right| \\
& \leq \frac{2}{\nu \mu^{2}}\|\varphi\|\left[\left\|\left(\chi_{\mu}(P)-11\right) Z_{\nu \mu} \varphi\right\|+\left\|\left(\chi_{-\mu}(P)-\mathbb{1}\right) Z_{-\nu \mu} \varphi\right\|\right]
\end{aligned}
$$


where we have used (4.15) in the last step. To bound the r.h.s. of 4.31) we will use the following identity, which is an easy consequence of 4.23:

$$
[F(P+s Q)-F(P)] \varphi=\frac{1}{2} \int_{0}^{s} \mathrm{~d} \tau[2(\nabla F)(P+\tau Q) \cdot Q-1(\Delta F)(P+\tau Q)] \varphi,
$$

where $F$ is any bounded function in $C^{\infty}\left(\mathbb{R}^{d}\right)$ with bounded derivatives.

Let $F \in C^{\infty}\left(\mathbb{R}^{d}\right)$ with $F \equiv 1$ near infinity, $F \equiv 0$ near 0 be such that

$$
F(Q)[\chi(Q)-\mathbb{1}]=\chi(Q)-\mathbb{1} .
$$

Then we have

$$
\left\|\left[\chi_{ \pm \mu}(P)-1\right] Z_{ \pm \nu \mu} \varphi\right\| \leq\left\|F\left(\frac{ \pm P}{\mu}\right) Z_{ \pm \nu \mu} \varphi\right\|=\left\|F\left(\frac{ \pm P}{\mu}+\nu Q\right) \varphi\right\|
$$

due to 4.23 . From 4.33 and the fact that

$$
F\left(\frac{ \pm P}{\mu}\right) \varphi=0, \quad \forall \mu \geq \mu_{0}
$$

we get for $\mu \geq \mu_{0}$

$\left|K_{\nu, \mu}(\varphi)\right| \leq \frac{2}{\nu \mu^{2}}\|\varphi\|\left\{\left\|\left[F\left(\frac{P}{\mu}+\nu Q\right)-F\left(\frac{P}{\mu}\right)\right] \varphi\right\|+\left\|\left[F\left(\frac{-P}{\mu}+\nu Q\right)-F\left(\frac{-P}{\mu}\right)\right] \varphi\right\|\right\}$

Moreover one has

$$
\left[F\left(\frac{ \pm P}{\mu}+\nu Q\right)-F\left(\frac{ \pm P}{\mu}\right)\right] \varphi=\frac{1}{2} \int_{0}^{\nu} \mathrm{d} \tau\left[2(\nabla F)\left(\frac{ \pm P}{\mu}+\tau Q\right) \cdot Q-1(\Delta F)\left(\frac{ \pm P}{\mu}+\tau Q\right)\right] \varphi
$$

due to 4.32). Therefore we have for $\mu \geq \mu_{0}$

$$
\left|K_{\nu, \mu}(\varphi)\right| \leq \text { Const. } \mu^{-2}\|\langle Q\rangle \varphi\|^{2} .
$$

The combination of (4.30) and (4.34) shows that $K_{\nu, \mu}(\varphi)$ is bounded uniformly in $\nu$ by a function in $\mathrm{L}^{1}([1,+\infty[, \mathrm{d} \mu)$.

\section{Acknowledgements}

R.T.d.A. thanks W. O. Amrein and P. Jacquet for their helpful remarks, and he also thanks the Swiss National Science Foundation for financial support.

\section{References}

[1] W. O. Amrein. Non-relativistic quantum dynamics, volume 2 of Math. Phys. Studies. D. Reidel Publishing Company, Dordrecht, 1981. 
[2] W. O. Amrein and M. B. Cibils. Global and Eisenbud-Wigner time delay in scattering theory. Helv. Phys. Acta, 60:481-500, 1987.

[3] W. O. Amrein, M. B. Cibils, and K. B. Sinha. Configuration space properties of the $S$-matrix and time delay in potential scattering. Ann. Inst. Henri Poincaré, 47:367$382,1987$.

[4] W. O. Amrein and P. Jacquet. Time delay for one-dimensional quantum systems with steplike potentials. In preparation.

[5] D. Bollé and T. A. Osborn. Time delay in $N$-body scattering. J. Math. Phys., 20:1121-1134, 1979.

[6] P. A. Martin. Scattering theory with dissipative interactions and time delay. Nuovo Cimento B, 30:217-238, 1975.

[7] P. A. Martin. Time delay in quantum scattering processes. Acta Phys. Austriaca Suppl., XXIII, pages 157-208, 1981.

[8] H. Narnhofer. Another definition for time delay. Phys. Rev. D, 22(10):2387-2390, 1980.

[9] M. Sassoli de Bianchi and P. A. Martin. On the definition of time delay in scattering theory. Helv. Phys. Acta, 65(8):1119-1126, 1992.

[10] F. T. Smith. Lifetime matrix in collision theory. Phys. Rev., 118:349-356, 1960.

[11] R. Tiedra de Aldecoa. Time delay and short-range scattering in quantum waveguides. Ann. Henri Poincaré, 7(1):105-124, 2006. 